Robin Fingerhut, Gerhard Herres, Jadran Vrabec

\title{
Thermodynamic factor of quaternary mixtures from Kirkwood-Buff integration
}

Journal article | Accepted manuscript (Postprint)

This version is available at https://doi.org/10.14279/depositonce-9121

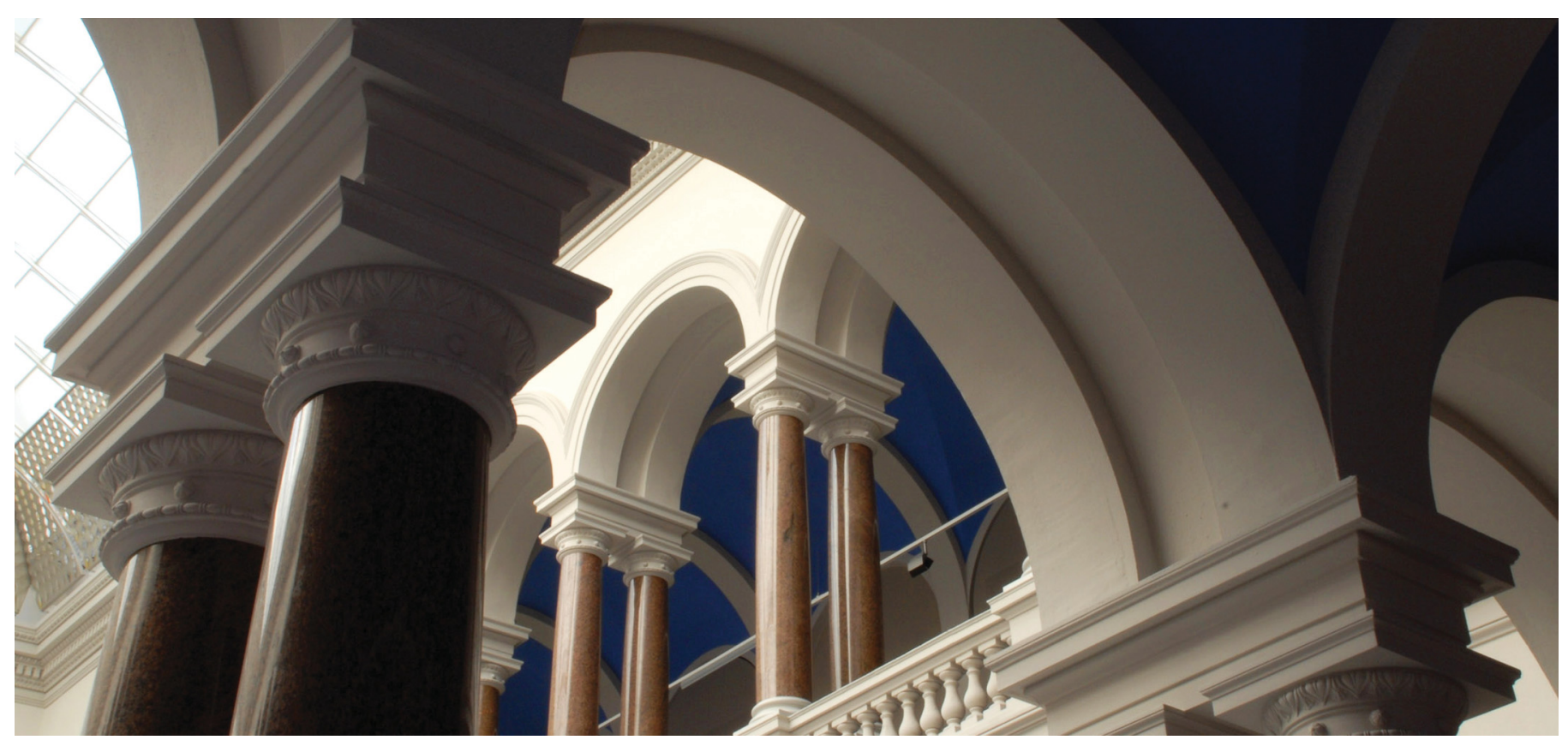

This is an Accepted Manuscript of an article published by Taylor \& Francis in Molecular Physics on 22 Jul 2019, available online: http://www.tandfonline.com/10.1080/00268976.2019.1643046

Fingerhut, R., Herres, G., \& Vrabec, J. (2019). Thermodynamic factor of quaternary mixtures from Kirkwood-Buff integration. Molecular Physics, 1-10. https://doi.org/10.1080/00268976.2019.1643046 


\title{
Thermodynamic factor of quaternary mixtures from Kirkwood-Buff integration
}

\author{
Robin Fingerhut ${ }^{\mathrm{a}}$, Gerhard Herres ${ }^{\mathrm{b}}$ and Jadran Vrabec ${ }^{\mathrm{a}}{ }^{*}$ \\ ${ }^{a}$ Thermodynamics and Process Engineering, Technical University Berlin, 10587 Berlin, \\ Germany, *E-Mail: vrabec@tu-berlin.de, \\ bThermodynamics and Energy Technology, University of Paderborn, 33098 Paderborn, \\ Germany
}

\section{ARTICLE HISTORY}

Compiled June 14, 2019

\begin{abstract}
Expressions for the thermodynamic factor matrix $\boldsymbol{\Gamma}$ of quaternary mixtures are derived in terms of Kirkwood-Buff integrals and implemented into the massivelyparallel simulation tool $m s 2$. To assess these expressions, a liquid-like supercritical quaternary Lennard-Jones mixture is sampled throughout its entire composition range, employing molecular dynamics in the canonical ensemble. Good agreement is found between numerical chemical potential derivatives and the results from the present Kirkwood-Buff integral expressions. Moreover, the limits of the thermodynamic factor matrix for pure, binary and ternary subsystems are discussed.
\end{abstract}

\section{KEYWORDS}

Kirkwood-Buff integration, thermodynamic factor, quaternary mixture, Lennard-Jones, molecular dynamics

\section{Introduction}

Accurate thermophysical data are essential for understanding natural processes and for designing technical applications [1]. Despite longstanding efforts in experimental thermodynamics, the available database is surprisingly sparse, particularly when it comes to higher order mixtures [2]. Utilizing suitable computational methods and high-performance computing, adequate prediction methods rapidly gain importance to overcome the insufficient thermodynamic data supply [3].

Equilibrium methods are prevalent for the design and optimization of separation processes, although they are not in equilibrium by definition. Addressing this conceptional shortcoming, rate-based methods that explicitly cover non-equilibrium dynamics 
are under strong development [4]. For their parametrization, however, not only equilibrium properties, but also transport coefficients are required, making thermodynamic data supply an even more pressing issue.

To describe mass transport in liquid mixtures, the thermodynamic factor $\boldsymbol{\Gamma}$ is important because it connects the Fick diffusion coefficient $D$ with the Maxwell-Stefan (MS) diffusion coefficient $D$. In case of a binary mixture, their relation is simply $D=\Gamma_{11} \cdot D$. The definition of this factor for a multi-component mixture is given by

$$
\Gamma_{i j}=\frac{x_{i}}{k T}\left(\frac{\partial \mu_{i}}{\partial x_{j}}\right)_{T, p, \Sigma}
$$

where $\mu_{i}$ is the chemical potential of component $i, x_{j}$ the mole fraction of component $j, T$ the temperature and $k$ the Boltzmann constant. This equation applies at $T, p=$ const., while the symbol $\Sigma$ indicates that $\sum_{i=1}^{n} x_{i}=1$ must be maintained during differentiation. Following the standard procedure, the partial differentiation of $\mu_{i}$ with respect to $x_{j}$ is carried out at constant mole fraction of all other components, except for the last [5].

In case of multi-component mixtures, this relation is given by $\boldsymbol{D}=\boldsymbol{B}^{-1} \boldsymbol{\Gamma}$, where matrix $\boldsymbol{B}$ is determined by the MS diffusion coefficient matrix $\boldsymbol{D}$ [5]. Matrices $\boldsymbol{D}$, $\boldsymbol{\Gamma}$ and $\boldsymbol{D}$ are of dimension $(n-1) \times(n-1)$ and only the latter one is symmetric. As a consequence, $\boldsymbol{\Gamma}$ connects four Fick with three MS diffusion coefficient elements for ternary mixtures, and nine Fick with six MS diffusion coefficient elements for quaternary mixtures. To characterize mass transport in higher order mixtures, matrix $\boldsymbol{D}$ asymptotically requires about twice as many parameter elements than matrix $\boldsymbol{D}$. This aspect is an important advantage of MS theory, which, however, rests on chemical potentials that are not accessible experimentally. On the contrary, when mass transport needs to be connected to laboratory work, where composition is treated explicitly, the Fick approach can be applied directly. To take advantage of both MS theory and Fick's law for a given scenario, the thermodynamic factor $\boldsymbol{\Gamma}$ is thus indispensable.

Due to its nature of being a derivative of the chemical potential $\mu_{i}$, the thermodynamic factor cannot be measured experimentally. Instead, this property has to be extracted from phase equilibrium data with the support of excess Gibbs energy models or equations of state. An elegant alternative route for sampling $\Gamma_{i j}$ is offered by molecular modeling and simulation combined with Kirkwood-Buff integration [6]. It should be noted that there are no other standard simulation methods available that can generally be applied.

Detailed information on the microscopic structure of fluids is provided by radial distribution functions (RDF) $g_{i j}$ sampled with many-body molecular simulations. On the basis of such data, that are closely related to local compositions, it is straightforward to analyze liquid solution properties by applying Kirkwood-Buff theory. However, Kirkwood-Buff integrals (KBI) are defined in the grand canonical $(\mu V T)$ ensemble 
only, which is hard to impose in simulations of dense liquid phases [7]. Instead, the standard procedure is to employ the canonical $(N V T)$ ensemble, which may lead to convergence problems. Numerous efforts have been made to counter these [7-13]. It was found that RDF corrections are crucial [7] and also that the extrapolation to the thermodynamic limit, where all ensemble types converge, is necessary $[9,10]$. These outcomes were confirmed by our recent study [14].

This work provides expressions for the thermodynamic factor matrix $\boldsymbol{\Gamma}$ of quaternary mixtures. For binary and ternary mixtures, this factor has already been derived and successfully evaluated $[5,6,15,16]$. Ben-Naim outlined the general formalism to determine the particle number derivative of the chemical potential from KBI [15]. This formalism was employed here to establish expressions of $\boldsymbol{\Gamma}$ for quaternary mixtures. They were implemented into the massively-parallel simulation tool $m s 2$ [17-19] and exemplarily applied to study a non-ideal Lennard-Jones (LJ) mixture. Furthermore, limits of the thermodynamic factor are discussed, i.e. the quaternary mixture towards its pure, binary and ternary subsystems.

\section{Methodology}

$m s 2$ samples $\mathrm{RDF} g_{i j}(r)$ for the molecules' center of mass, which are the essential input for KBI. Our recent study [14], which is in good agreement with others [7, 12], has confirmed that RDF corrections are required in this context. The RDF correction proposed by Ganguly and van der Vegt [20] was found to be most adequate and takes into account that molecules may underlie excess or depletion phenomena so that it was applied in this work throughout.

As mentioned above, it is challenging to impose the $\mu V T$ ensemble for dense liquid phase simulations. Facing this issue, Krüger et al. [13] developed an integral truncation and correction, in order to apply KBI to $N V T$ ensemble simulation data. Their mathematical procedure considers that finite simulation volumes, as sampled by the $N V T$ ensemble, are embedded in a larger reservoir. In this way, the exchange of mass and energy of the explicitly sampled volume with its surrounding is accounted for. This KBI truncation and correction [13] was employed here because of its success [14]. Moreover, it was found that the extrapolation to the thermodynamic limit $V \rightarrow \infty$ [13] is essential $[9,10,14]$. Therefore, a recently proposed KBI approximation for the thermodynamic limit [9] was used here throughout due to its faster convergence than the standard analytical form [13].

Expressions for $\boldsymbol{\Gamma}$ calculated from KBI for binary and ternary mixtures are provided in the literature $[5,6,15,16]$. In case of a binary mixture, it is simply the scalar

$$
\Gamma_{11}=1-\frac{x_{1} \rho_{2} \Delta_{12}}{1+x_{1} \rho_{2} \Delta_{12}}
$$


where $\rho_{2}=x_{2} \rho$ with mixture density $\rho, \Delta_{12}=G_{11}+G_{22}-2 G_{12}$ and $G_{i j}$ are KBI with component indices $i$ and $j$. In the ternary case, $\boldsymbol{\Gamma}$ is a $(2 \times 2)$ matrix [5], cf. Appendix B.

The general formalism for obtaining chemical potential derivatives from KBI was outlined by Ben-Naim [15]. The derivative of the chemical potential $\mu_{i}$ with respect to the particle number $N_{j}$ is given by

$$
\left(\frac{\partial \mu_{i}}{\partial N_{j}}\right)_{T, p, N_{j}^{\prime}}=\frac{k T}{V|\boldsymbol{B}|} \frac{\sum_{a=1}^{n} \sum_{b=1}^{n} \rho_{a} \rho_{b}\left(B^{i j} B^{a b}-B^{a i} B^{b j}\right)}{\sum_{a=1}^{n} \sum_{b=1}^{n} \rho_{a} \rho_{b} B^{a b}}
$$

with volume $V$, component indices $i, j, a, b$ and total number of components in the mixture $n$. Note that $N_{j}^{\prime}$ indicates that all particle numbers $N_{i}$ are kept constant, except for $N_{j}$. Eq. (3) gives evidence that KBI are defined for open systems, like the $\mu V T$ ensemble, because the total number of molecules is not constant. The $(n \times$ $n)$ matrix $\boldsymbol{B}$ consists of the elements $B_{i j}=\rho_{i} \rho_{j} G_{i j}+\rho_{i} \delta_{i j}$, where $\delta_{i j}$ denotes the Kronecker delta. In eq. (3), $B^{i j}$ are derivatives of the determinant $|\boldsymbol{B}|$ with respect to $B_{i j}$, i.e. $B^{i j}=\partial|\boldsymbol{B}| / \partial B_{i j}[15]$. Note that matrix $\boldsymbol{B}$ is symmetric because $g_{i j}=g_{j i}$ and thus $G_{i j}=G_{j i}$.

The asymmetric $(3 \times 3)$ thermodynamic factor matrix $\boldsymbol{\Gamma}$ can be determined for quaternary mixtures based on eq. (3). Such a mixture has four mole fractions $x_{1}$, $x_{2}, x_{3}$ and $x_{4}$, where the first three were considered to be independent. The matrix elements are defined by eq. (1) so that the relation between eqs. (1) and (3) needs to be written out, which is presented for a quaternary mixture in Appendix C.

\section{Molecular model}

Expressions for $\boldsymbol{\Gamma}$ were assessed for a quaternary LJ mixture under liquid-like supercritical conditions. The LJ size parameter of all four components was specified to be identical $\sigma_{1}=\sigma_{2}=\sigma_{3}=\sigma_{4}$ to ensure statistically sound chemical potential $\mu_{i}$ sampling with Widom's test particle insertion [21]. In order to truly distinguish the components, the LJ energy parameters were assumed to be $\varepsilon_{2} / \varepsilon_{1}=5 / 6, \varepsilon_{3} / \varepsilon_{1}=25 / 36$ and $\varepsilon_{4} / \varepsilon_{1}=125 / 216$. Due to these moderate intermolecular interaction energy differences, a significant non-ideality had to be introduced by the modified Berthelot combination rule [22] $\varepsilon_{i j}=\xi \sqrt{\varepsilon_{i} \varepsilon_{j}}$ with all six binary parameters set to $\xi=1.5$. By defining the value of $\xi$ to be far above unity, a mixture with a maximum boiling azeotrope for all of its binary subsystems is compounded.

This quaternary mixture was studied at constant temperature $k T / \varepsilon_{1}=2.2$ and pressure $p \sigma_{1}^{3} / \varepsilon_{1}=4$. Because of $k T_{c} / \varepsilon_{i}=1.32$ [23], the chosen temperature is supercritical for all four components and the specified very high pressure led to pure 


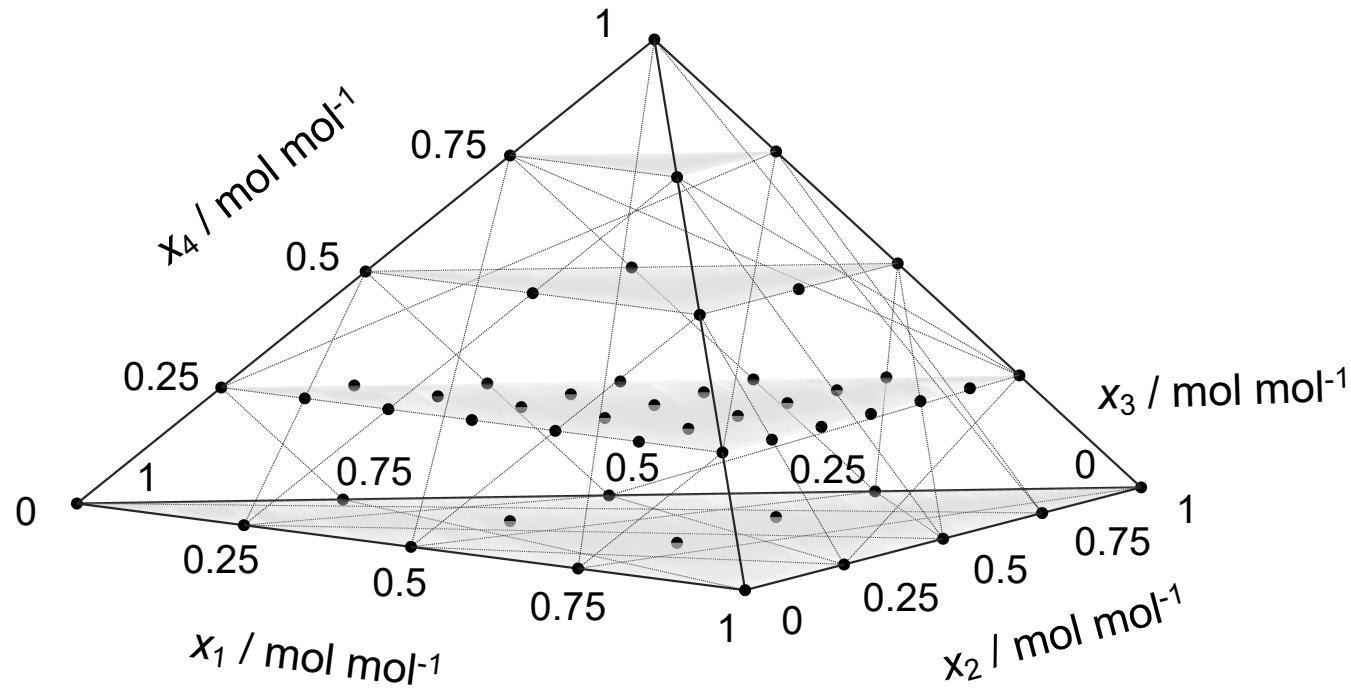

Figure 1. Studied compositions of the quaternary LJ mixture are indicated by black circles, while the grey planes are guides to the eye.

fluid densities $\rho$ larger than their critical one $\rho_{c} \sigma_{i}^{3}=0.31$ [23], i.e. $\rho_{1} \sigma_{1}^{3}=0.721$, $\rho_{2} \sigma_{2}^{3}=0.703, \rho_{3} \sigma_{3}^{3}=0.688$ and $\rho_{4} \sigma_{4}^{3}=0.678$. In this way, it was ensured that the mixture was liquid-like supercritical throughout the entire studied composition range, which is illustrated in figure 1.

NVT ensemble molecular dynamics (MD) simulations were carried out with $N=$ 8000 particles that were equilibrated by 100 Monte Carlo cycles and $8 \cdot 10^{5} \mathrm{MD}$ time steps. To sample KBI and thus $\boldsymbol{\Gamma}$, production runs were always performed for a period of $4 \cdot 10^{7}$ time steps. Beforehand, isobaric-isothermal $(N p T)$ ensemble MD simulations were conducted to determine the mixture density. Newton's equations of motion were solved numerically by applying the Gear predictor-corrector integrator [24] with a time step of $\Delta t /\left(\sigma_{1} \sqrt{m_{1} / \varepsilon_{1}}\right)=0.0003$ for all simulations. Velocities were isokinetically rescaled to maintain the specified temperature. The cutoff radius was set to $4 \sigma_{1}$ and the long-range interactions were corrected with the usual analytic mean-field equations. Sampled state points are shown in figure 1, where the vertices indicate the pure components, the edges are the binary subsystems and the pyramid surfaces are the ternary subsystems. Only state points inside the pyramid are truly quaternary mixtures so that the plane at $x_{4}=0.25 \mathrm{~mol} \mathrm{~mol}^{-1}$ was studied with a finer discretization.

\section{Results}

Two quaternary state points were selected to validate the present $\boldsymbol{\Gamma}$ expression in detail. For this purpose, $\boldsymbol{\Gamma}$ was calculated from approximative chemical potential deriva- 


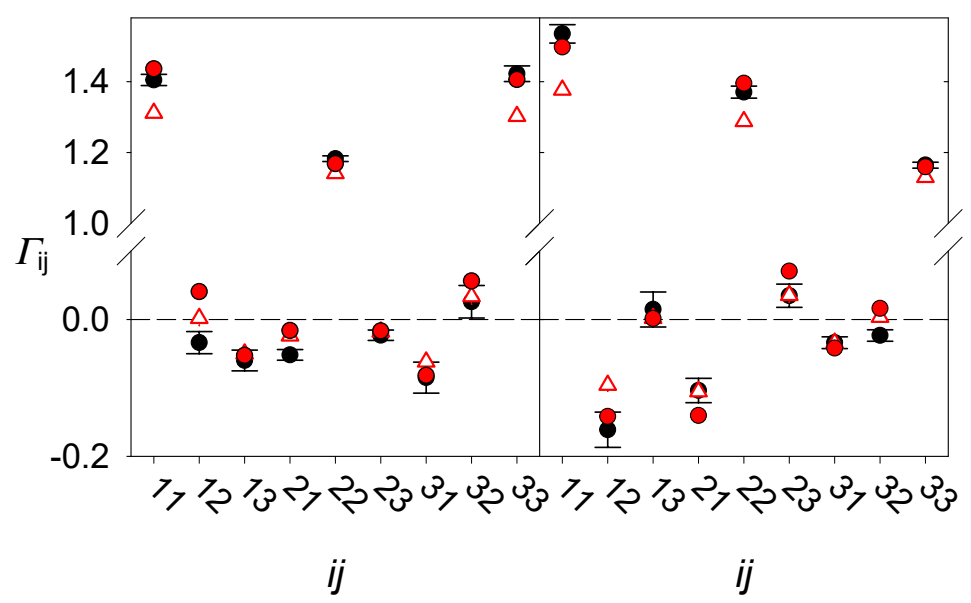

Figure 2. Thermodynamic factor $\boldsymbol{\Gamma}$ at two quaternary state points; black circles: $\boldsymbol{\Gamma}$ based on numerical chemical potential derivatives sampled with Widom's test particle insertion [21]; red circles/triangles: $\boldsymbol{\Gamma}$ calculated with the present expressions for quaternary mixtures based on extrapolated KBI [9]/non-extrapolated KBI [13] (statistical uncertainties are within symbol size); left: $x_{1}=0.25 \mathrm{~mol} \mathrm{~mol}^{-1}, x_{2}=0.125 \mathrm{~mol} \mathrm{~mol}^{-1}$ and $x_{3}=0.375 \mathrm{~mol} \mathrm{~mol}^{-1}$; right: $x_{1}=0.375 \mathrm{~mol} \mathrm{~mol}^{-1}, x_{2}=0.25 \mathrm{~mol} \mathrm{~mol}^{-1}$ and $x_{3}=0.125 \mathrm{~mol} \mathrm{~mol}^{-1}$.

tives and compared with $\boldsymbol{\Gamma}$ from KBI. For the former, the chemical potential $\mu_{i}$ was sampled with Widom's test particle insertion [21] using NVT ensemble MD simulations which were performed over $2 \cdot 10^{7}$ time steps.

To obtain the thermodynamic factor by numerical derivation, $\mu_{i}$ data were calculated at two different mole fractions near the target one $x_{1}, x_{2}$ and $x_{3}$, i.e. $x_{i}=x_{i} \pm 0.01 \mathrm{~mol} \mathrm{~mol}^{-1}$, respectively, while $x_{4}$ ensured $\sum_{i} x_{i}=1$. Figure 2 shows a comparison for two quaternary state points. Because of the mixtures' strong nonideality, the main elements of $\boldsymbol{\Gamma}$ are considerably larger than unity. From the agreement of the nine matrix elements, that is almost throughout within the small statistical uncertainties, it can be concluded that the present $\boldsymbol{\Gamma}$ expressions based on extrapolated KBI [9] are appropriate. Numerical data for $\boldsymbol{\Gamma}$ from extrapolated and non-extrapolated KBI for the entire composition range can be found in the Supporting Information.

\section{Graphical overview}

The liquid-like supercritical LJ mixture introduced above was studied over the entire composition range to get an impression of $\boldsymbol{\Gamma}$. The ternary plane at $x_{4}=0$ was evaluated with a discretization of $\Delta x=0.25 \mathrm{~mol} \mathrm{~mol}^{-1}$, cf. figure 1 . This plane contains three pure component systems at the vertices, nine binary state points along the edges and three ternary state points inside the plane. To determine the matrix elements of $\boldsymbol{\Gamma}$ for the entire plane at $x_{4}=0$, it is crucial to investigate the limits, i.e. the ternary mixture towards its pure or binary subsystems. These limits are discussed in Appendix B, where it is shown that some elements of $\boldsymbol{\Gamma}$ cannot be sampled with KBI 
in a statistically sound way because of uncertain $\operatorname{RDF} g_{i j}$ data at infinite dilution.

A composition slice of the quaternary LJ mixture was studied for $x_{4}=$ $0.25 \mathrm{~mol} \mathrm{~mol}^{-1}$ with $\Delta x=0.125 \mathrm{~mol} \mathrm{~mol}^{-1}$, cf. figure 1 . Its three vertices are binary subsystems, the three edges are ternaries, only state points inside the plane are truly quaternary mixtures. Like the ternary case, some limits of $\boldsymbol{\Gamma}$ cannot be sampled in a statistically sound way with KBI, cf. Appendix C.

To obtain a smooth visual representation, the Wilson model [25] was fitted to the present $\boldsymbol{\Gamma}$ simulation data based on extrapolated KBI [9]. Its parameters are listed in the Supporting Information and a satisfying agreement between $\boldsymbol{\Gamma}$ from the Wilson model and $\boldsymbol{\Gamma}$ from KBI was found. Utilizing the Wilson model, an analysis of the ternary and quaternary limits was feasible.

Figure 3 shows $\boldsymbol{\Gamma}$ based on the Wilson model for the ternary subsystem at $x_{4}=0$. Therein, the surfaces depict the matrix elements of $\boldsymbol{\Gamma}$. The main elements $\Gamma_{11}$ and $\Gamma_{22}$ reach values from unity to around 1.7 , whereas the cross elements $\Gamma_{12}$ and $\Gamma_{21}$ attain values between around \pm 0.5 . Main and cross elements clearly differ from each other due to their numerical range and curvature. Moreover, this figure confirms the limits of $\boldsymbol{\Gamma}$ which are given in Appendix B for the ternary case. The first limit, i.e. ternary mixture towards its pure fluids is $\Gamma_{11}=\Gamma_{22}=1$ and $\Gamma_{12}=\Gamma_{21}=0$ at the three vertices. The second limit, i.e. ternary mixture towards its binary subsystems, is shown at the edges. Exemplarily, for $x_{1} \rightarrow 0$, the main element $\Gamma_{11}=1$ and $\Gamma_{22}$ is the binary thermodynamic factor of the subsystem $2+3$. The cross element $\Gamma_{12}=0$, whereas $\Gamma_{21}$ is neither zero nor unity. Sampling $\Gamma_{21}$ in a statistically sound way with KBI becomes challenging due to uncertain RDF $g_{12}$ and $g_{13}$ at infinite dilution of component 1, cf. Appendix B.

Figure 4 depicts $\boldsymbol{\Gamma}$ from the Wilson model for the quaternary LJ mixture at $x_{4}=0.25 \mathrm{~mol} \mathrm{~mol}^{-1}$. Again, the main elements are distinctly different from the cross elements because $\Gamma_{11}, \Gamma_{22}$ and $\Gamma_{33}$ attain values between unity and around 1.5, whereas the cross elements are around \pm 0.2 . Furthermore, main element and cross element surfaces have different curvatures. The first limit, i.e. quaternary mixture towards the pure fluids, cannot be seen in this figure, but the second limit, i.e. quaternary mixture towards its binary subsystems is shown at the vertices. For instance, in case of $x_{1}+x_{2} \rightarrow 0$, the quaternary system converges towards the binary subsystem $3+4$. In this case, the main elements are $\Gamma_{11}=1, \Gamma_{22}=1$ and $\Gamma_{33}$ is the binary thermodynamic factor. The cross elements $\Gamma_{31} \neq 0$ and $\Gamma_{32} \neq 0$ cannot be sampled in a statistically sound way because of uncertain RDF $g_{13}, g_{14}$ for the former and $g_{23}, g_{24}$ for the latter at infinite dilution of components 1 and 2 . All other cross elements of $\boldsymbol{\Gamma}$ are zero. The third limit, i.e. the quaternary mixture towards its ternary subsystems is visible at the edges of the plane at $x_{4}=0.25 \mathrm{~mol} \mathrm{~mol}^{-1}$. Exemplarily, for $x_{1} \rightarrow 0$, the quaternary mixture converges towards the ternary subsystem $2+3+4$. Consequently, $\Gamma_{11}=1$, $\Gamma_{12}=\Gamma_{13}=0, \Gamma_{21} \neq 0, \Gamma_{31} \neq 0$ and the remaining matrix elements of $\boldsymbol{\Gamma}$ correspond to the ternary $(2 \times 2) \Gamma$ matrix. Again, the elements $\Gamma_{21} \neq 0$ and $\Gamma_{31} \neq 0$ cannot be 


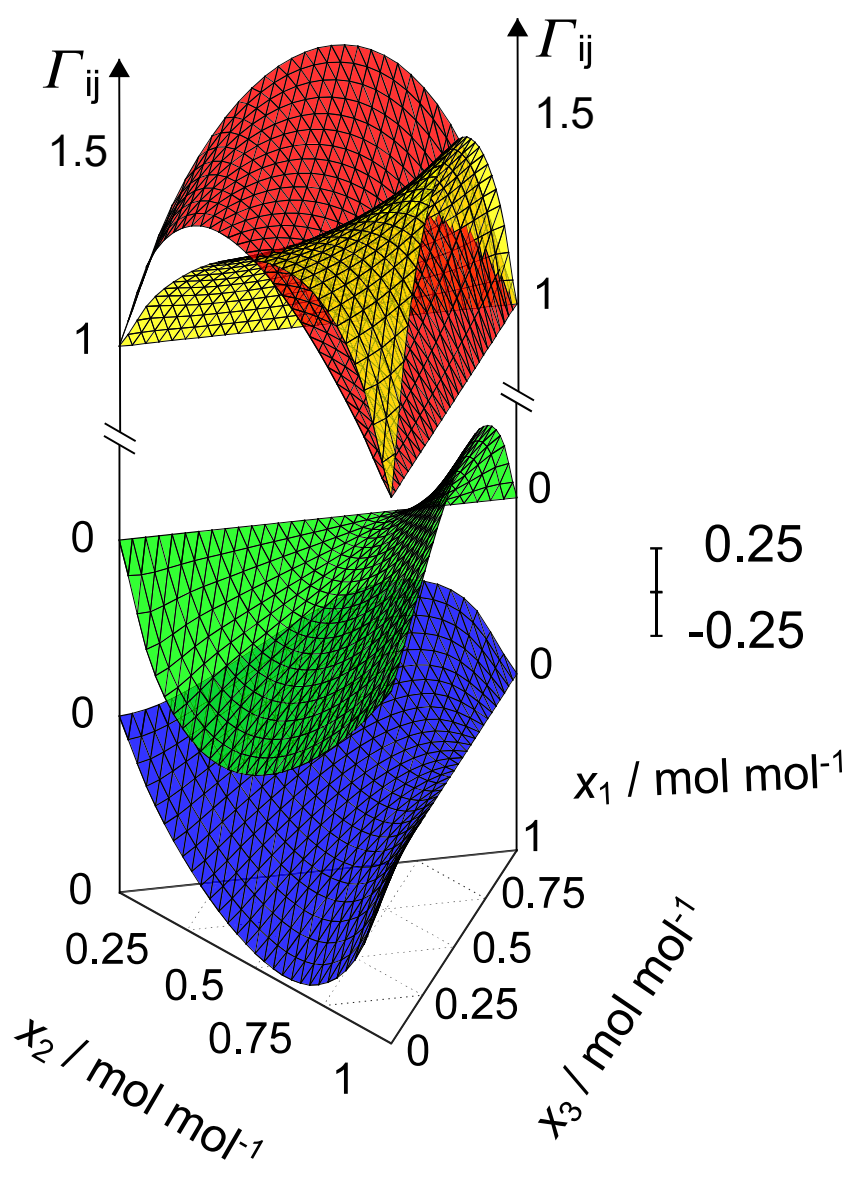

Figure 3. Thermodynamic factor $\boldsymbol{\Gamma}$ of the ternary subsystem at $x_{4}=0$ based on the Wilson model [25] fitted to $\boldsymbol{\Gamma}$ data determined from extrapolated KBI [9]; red surface: $\Gamma_{11} ;$ yellow surface: $\Gamma_{22}$; blue surface: $\Gamma_{12} ;$ green surface: $\Gamma_{21}$; the cross elements of $\boldsymbol{\Gamma}$ were shifted for visibility reasons, while zeros at the vertical axes indicate their limiting values. 
sampled in a statistically sound way with KBI. Details on these limits are given in Appendix C.

\section{Conclusion}

Describing mass transport coefficients of liquid mixtures is an important topic in modern process engineering, however, the limited data supply poses a serious challenge, particularly when it comes to higher order mixtures. To connect the Fick and MaxwellStefan diffusion formalisms, the thermodynamic factor $\boldsymbol{\Gamma}$ is crucial. However, this factor cannot be measured experimentally because it is a derivative of the chemical potential $\mu_{i}$. Kirkwood-Buff integration [6] offers an elegant access to sample this property by molecular simulation.

Up to now, only $\boldsymbol{\Gamma}$ expressions for binary and ternary mixtures were available $[5,6$, 15, 16]. Extending these works, expressions for $\boldsymbol{\Gamma}$ of quaternary mixtures were derived in this work. For this purpose, Ben-Naim's [15] general form of the chemical potential derivative in terms of KBI was applied. The equations for matrix $\boldsymbol{\Gamma}$ given in Appendix C were implemented into the massively-parallel simulation tool $m s 2$ [17-19].

A liquid-like supercritical quaternary non-ideal LJ mixture was simulated over the entire composition range to study the present $\boldsymbol{\Gamma}$ expressions. Extrapolated KBI [9] were sampled by $N V T$ ensemble MD simulations based on the RDF correction by Ganguly and van der Vegt [20]. A detailed comparison based on numerical chemical potential derivatives and extrapolated KBI showed a good agreement. Limits of $\boldsymbol{\Gamma}$ for binary, ternary and quaternary mixtures were discussed.

Instead of sampling chemical potentials, KBI provides an elegant avenue to determine $\boldsymbol{\Gamma}$ for quaternary mixtures that can efficiently be implemented and parallelized. The thermodynamic factor $\boldsymbol{\Gamma}$ of mixtures with more than four components are important too, but the length of according expressions would escalate further. For their derivation and implementation, automatic procedures may be required for higher order mixtures.

\section{Acknowledgements}

This research was funded by BMBF under the grant 01IH16008 "TaLPas: Task-basierte Lastverteilung und Auto-Tuning in der Partikelsimulation" and computational support was given by the High Performance Computing Center Stuttgart (HLRS) under the grant MMHBF2. Furthermore, we gratefully acknowledge the Paderborn Center for Parallel Computing $\left(\mathrm{PC}^{2}\right)$ for the generous allocation of computer time on the OCuLUS and Noctua clusters. 


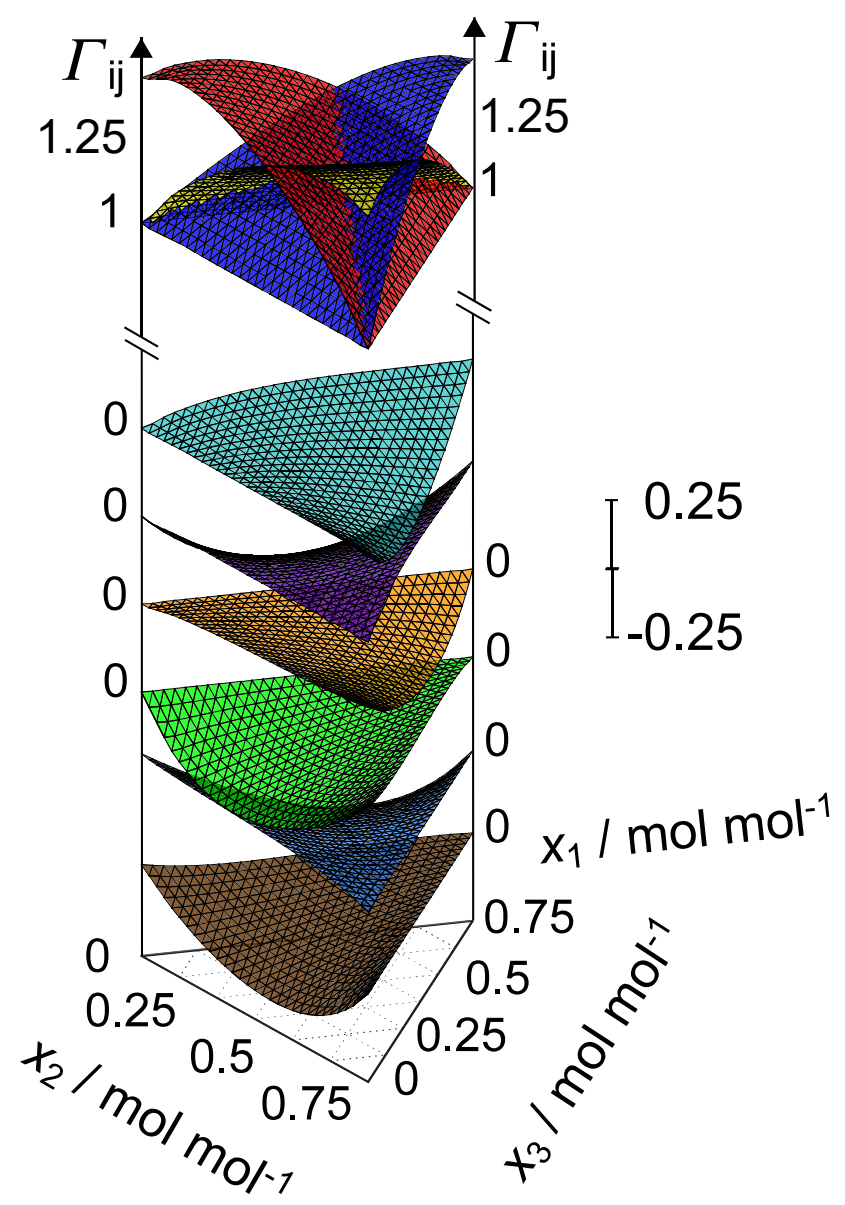

Figure 4. Thermodynamic factor $\boldsymbol{\Gamma}$ of the quaternary system at $x_{4}=0.25 \mathrm{~mol} \mathrm{~mol}^{-1}$ based on the Wilson model [25] fitted to $\Gamma$ data determined from extrapolated KBI [9]; red surface: $\Gamma_{11}$; yellow surface: $\Gamma_{22}$; blue surface: $\Gamma_{33}$; brown surface: $\Gamma_{12}$; light blue surface: $\Gamma_{13}$; green surface: $\Gamma_{21}$; orange surface: $\Gamma_{23} ;$ purple surface: $\Gamma_{31}$; cyan surface: $\Gamma_{32}$; the cross elements of $\boldsymbol{\Gamma}$ were shifted for visibility reasons, while zeros at the vertical axes indicate their limiting values. 


\section{Funding}

German Federal Ministry of Education and Research (BMBF).

\section{References}

\section{References}

[1] E. Hendriks, G.M. Kontogeorgis, R. Dohrn, J.C. de Hemptinne, I.G. Economou, L.F. Zilnik and V. Vesovic, Ind. Eng. Chem. Res. 49, 11131-11141 (2010).

[2] K.E. Gubbins and J.D. Moore, Ind. Eng. Chem. Res. 49, 3026-3046 (2010).

[3] E.J. Maginn, AIChE J. 55, 1304-1310 (2009).

[4] E.Y. Kenig and S. Blagov, Modeling of distillation processes in Distillation: Fundamentals and Principles, edited by A. Gorak and E. Sorensen (Elsevier, London, 2014).

[5] X. Liu, A. Martín-Calvo, E. McGarrity, S.K. Schnell, S. Calero, J.M. Simon, D. Bedeaux, S. Kjelstrup, A. Bardow and T.J.H. Vlugt, Ind. Eng. Chem. Res. 51, 10247-10258 (2012).

[6] J.G. Kirkwood and F.P. Buff, J. Chem. Phys. 19, 774-778 (1951).

[7] J. Milzetti, D. Nayar and N.F.A. van der Vegt, J. Phys. Chem. B 122, 5515-5526 (2018).

[8] M. Heidari, K. Kremer, R. Potestio and R. Cortes-Huerto, Entropy 20, 222-238 (2018).

[9] P. Krüger and T.J.H. Vlugt, Phys. Rev. E. 97, 051301 (2018).

[10] S.K. Schnell, X. Liu, J.M. Simon, A. Bardow, D. Bedeaux, T.J.H. Vlugt and S. Kjelstrup, J. Phys. Chem. B 115, 10911-10918 (2011).

[11] A.A. Galata, S.D. Anogiannakis and D.N. Theodorou, Fluid Phase Equilib. 470, 25-37 (2018).

[12] N. Dawass, P. Krüger, S.K. Schnell, D. Bedeaux, S. Kjelstrup, J.M. Simon and T.J.H. Vlugt, Mol. Simul. 44, 599-61 (2018).

[13] P. Krüger, S.K. Schnell, D. Bedeaux, S. Kjelstrup, T.J.H. Vlugt and J.M. Simon, J. Phys. Chem. Lett. 4, 235-238 (2013).

[14] R. Fingerhut and J. Vrabec, Fluid Phase Equilib. 485, 270-281 (2019).

[15] A. Ben-Naim, Molecular Theory of Solutions (Oxford University Press, Oxford, 2006).

[16] E. Ruckenstein and I. Shulgin, Fluid Phase Equilib. 180, 345-359 (2001).

[17] S. Deublein, B. Eckl, J. Stoll, S.V. Lishchuk, G. Guevara-Carrion, C.W. Glass, T. Merker, M. Bernreuther, H. Hasse and J. Vrabec, Comput. Phys. Commun. 182, 2350-2367 (2011).

[18] C.W. Glass, S. Reiser, G. Rutkai, S. Deublein, A. Köster, G. Guevara-Carrion, A. Wafai, M. Horsch, M. Bernreuther, T. Windmann, H. Hasse and J. Vrabec, Comp. Phys. Commun. 185, 3302-3306 (2014).

[19] G. Rutkai, A. Köster, G. Guevara-Carrion, T. Janzen, M. Schappals, C.W. Glass, M. Bernreuther, A. Wafai, S. Stephan, M. Kohns, S. Reiser, S. Deublein, M. Horsch, H. Hasse and J. Vrabec, Comp. Phys. Commun. 221, 343-351 (2017).

[20] P. Ganguly and N.F.A. van der Vegt, J. Chem. Theor. Comput. 9, 1347-1355 (2013).

[21] B. Widom, J. Chem. Phys. 39, 2808-2812 (1963).

[22] D. Berthelot, Compt. Rend. Acad. Sci. Paris 126, 1703-1855 (1898).

[23] M. Thol, G. Rutkai, A. Köster, R. Lustig, R. Span and J. Vrabec, J. Phys. Chem. Ref. Data 45, 023101 (2016). 
[24] D.J. Allen and M.P. Tildesley, Computer Simulation of Liquids (Oxford University Press, Oxford, 1989).

[25] G.M. Wilson, J. Am. Chem. Soc. 86, 127-130 (1964).

[26] D.A. Jonah and H.D. Cochran, Fluid Phase Equilib. 92, 107-137 (1994).

\section{Appendix A. Thermodynamic factor of binary mixtures}

For a binary mixture, the thermodynamic factor matrix $\boldsymbol{\Gamma}$ is simply a scalar $[6,15]$

$$
\Gamma_{11}=1-\frac{x_{1} \rho_{2} \Delta_{12}}{1+x_{1} \rho_{2} \Delta_{12}}
$$

where $\rho_{2}=x_{2} \rho$ with the mixture density $\rho, \Delta_{12}=G_{11}+G_{22}-2 G_{12}$ and $G_{i j}$ are KBI with component indices $i$ and $j$.

The pure fluid limits are straightforward

$$
\lim _{x_{1} \rightarrow 0} \Gamma_{11}=\lim _{x_{2} \rightarrow 0} \Gamma_{11}=1
$$

\section{Appendix B. Thermodynamic factor of ternary mixtures}

The thermodynamic factor $\boldsymbol{\Gamma}$ of a ternary mixture is a $(2 \times 2)$ matrix. To lighten notation, the following auxiliary expressions were defined $[5,15,16]$

$$
\begin{gathered}
\Delta_{i j}=G_{i i}+G_{j j}-2 G_{i j}, i \neq j, \\
\eta=\rho_{1}+\rho_{2}+\rho_{3}+\rho_{1} \rho_{2} \Delta_{12}+\rho_{1} \rho_{3} \Delta_{13}+\rho_{2} \rho_{3} \Delta_{23} \\
-\frac{1}{4} \rho_{1} \rho_{2} \rho_{3}\left(\Delta_{12}^{2}+\Delta_{13}^{2}+\Delta_{23}^{2}-2\left[\Delta_{13} \Delta_{23}+\Delta_{12} \Delta_{13}+\Delta_{12} \Delta_{23}\right]\right),
\end{gathered}
$$

with $\rho_{i}=x_{i} \rho$ and mixture density $\rho$. The elements of $\boldsymbol{\Gamma}$ are $[5,15,16]$

$$
\Gamma_{11}=\frac{1}{\eta}\left(\rho_{1}+\rho_{2}+\rho_{3}+\rho_{1} \rho_{2}\left[G_{22}-G_{12}+G_{13}-G_{23}\right]+\rho_{2} \rho_{3} \Delta_{23}\right),
$$




$$
\begin{aligned}
& \Gamma_{12}=\frac{\rho_{1}}{\eta}\left(\rho_{2}\left[G_{22}-G_{12}+G_{13}-G_{23}\right]-\rho_{3}\left[G_{33}-G_{13}+G_{12}-G_{23}\right]\right), \\
& \Gamma_{21}=\frac{\rho_{2}}{\eta}\left(\rho_{1}\left[G_{11}-G_{12}+G_{23}-G_{13}\right]-\rho_{3}\left[G_{33}-G_{13}+G_{12}-G_{23}\right]\right), \\
& \Gamma_{22}=\frac{1}{\eta}\left(\rho_{1}+\rho_{2}+\rho_{3}+\rho_{1} \rho_{2}\left[G_{11}-G_{12}-G_{13}+G_{23}\right]+\rho_{1} \rho_{3} \Delta_{13}\right) .
\end{aligned}
$$

The pure fluid limits are equivalent to the binary case, except for their dimension

$$
\lim _{\substack{x_{1} \rightarrow 0 \\
x_{2} \rightarrow 0}} \boldsymbol{\Gamma}=\lim _{\substack{x_{1} \rightarrow 0 \\
x_{3} \rightarrow 0}} \boldsymbol{\Gamma}=\lim _{\substack{x_{2} \rightarrow 0 \\
x_{3} \rightarrow 0}} \boldsymbol{\Gamma}=\left(\begin{array}{ll}
1 & 0 \\
0 & 1
\end{array}\right)
$$

The second limit, i.e. the ternary mixture towards its binary subsystem, is

$$
\begin{aligned}
& \lim _{x_{1} \rightarrow 0} \boldsymbol{\Gamma}=\left(\begin{array}{cc}
1 & 0 \\
\Gamma_{21} & \Gamma_{B}
\end{array}\right), \\
& \lim _{x_{2} \rightarrow 0} \boldsymbol{\Gamma}=\left(\begin{array}{cc}
\Gamma_{B} & \Gamma_{12} \\
0 & 1
\end{array}\right), \\
& \lim _{x_{3} \rightarrow 0} \boldsymbol{\Gamma}=\left(\begin{array}{cc}
\Gamma_{B}+\Gamma_{12} & \Gamma_{12} \\
\Gamma_{21} & \Gamma_{B}+\Gamma_{21}
\end{array}\right) .
\end{aligned}
$$

Looking at eq. (B8) for $x_{1} \rightarrow 0$, the main elements are $\Gamma_{11}=1$ and $\Gamma_{B}$, where the latter is the thermodynamic factor of the binary subsystem $2+3$ given by eq. (A1). The cross element $\Gamma_{12}$ is zero, whereas $\Gamma_{21}$ is neither zero nor unity. Due to $x_{1} \rightarrow 0$, parts of eq. (B5) vanish, but a statistically sound sampling of $\Gamma_{21}$ with KBI is not straightforward because of RDF $g_{12}$ and $g_{13}$ are needed at infinite dilution of component 1 . The other cases, $x_{2} \rightarrow 0$ and $x_{3} \rightarrow 0$, are analogous. 


\section{Appendix C. Thermodynamic factor of quaternary mixtures}

A quaternary mixture has four mole fractions $x_{1}, x_{2}, x_{3}$ and $x_{4}$. Here, the first three were taken as independent. The $(3 \times 3)$ thermodynamic factor matrix of a quaternary mixture $\boldsymbol{\Gamma}$ is not symmetric and its elements are defined by eq. (1). To calculate its elements, the relation between $\left(\partial \mu_{i} / \partial x_{j}\right)_{T, p, \Sigma}$ and $\left(\partial \mu_{i} / \partial N_{j}\right)_{T, p, N_{j}^{\prime}}$ is needed, cf. eqs. (1) and (3). The latter derivative was presented in the context of KBI for mixtures with an arbitrary number of components by Ben-Naim [15]. Based on the work of Jonah and Cochran [26], the relation for the quaternary case is

$$
\begin{aligned}
&\left(\frac{\partial \mu_{1}}{\partial x_{1}}\right)_{T, p, \Sigma}=N \cdot\left[\left(\frac{\partial \mu_{1}}{\partial N_{1}}\right)_{T, p, N_{1}^{\prime}}-\left(\frac{\partial \mu_{1}}{\partial N_{4}}\right)_{T, p, N_{4}^{\prime}}\right], \\
&\left(\frac{\partial \mu_{2}}{\partial x_{1}}\right)_{T, p, \Sigma}=N \cdot\left[\left(\frac{\partial \mu_{2}}{\partial N_{1}}\right)_{T, p, N_{1}^{\prime}}-\left(\frac{\partial \mu_{2}}{\partial N_{4}}\right)_{T, p, N_{4}^{\prime}}\right], \\
&\left(\frac{\partial \mu_{3}}{\partial x_{1}}\right)_{T, p, \Sigma}=N \cdot\left[\left(\frac{\partial \mu_{3}}{\partial N_{1}}\right)_{T, p, N_{1}^{\prime}}-\left(\frac{\partial \mu_{3}}{\partial N_{4}}\right)_{T, p, N_{4}^{\prime}}\right], \\
&\left(\frac{\partial \mu_{1}}{\partial x_{2}}\right)_{T, p, \Sigma}=N \cdot\left[\left(\frac{\partial \mu_{1}}{\partial N_{2}}\right)_{T, p, N_{2}^{\prime}}-\left(\frac{\partial \mu_{1}}{\partial N_{4}}\right)_{T, p, N_{4}^{\prime}}\right], \\
&\left(\frac{\partial \mu_{1}}{\partial x_{3}}\right)_{T, p, \Sigma}=N \cdot\left[\left(\frac{\partial \mu_{1}}{\partial N_{3}}\right)_{T, p, N_{3}^{\prime}}-\left(\frac{\partial \mu_{1}}{\partial N_{4}}\right)_{T, p, N_{4}^{\prime}}\right], \\
&\left(\frac{\partial \mu_{2}}{\partial x_{2}}\right)_{T, p, \Sigma}=N \cdot\left[\left(\frac{\partial \mu_{2}}{\partial N_{2}}\right)_{T, p, N_{2}^{\prime}}-\left(\frac{\partial \mu_{2}}{\partial N_{4}}\right)_{T, p, N_{4}^{\prime}}\right], \\
&=N \cdot\left[\left(\frac{\partial \mu_{3}}{\partial N_{2}}\right)_{T, p, N_{2}^{\prime}}-\left(\frac{\partial \mu_{3}}{\partial N_{4}}\right)_{T, p, N_{4}^{\prime}}\right],
\end{aligned}
$$




$$
\begin{aligned}
& \left(\frac{\partial \mu_{2}}{\partial x_{3}}\right)_{T, p, \Sigma}=N \cdot\left[\left(\frac{\partial \mu_{2}}{\partial N_{3}}\right)_{T, p, N_{3}^{\prime}}-\left(\frac{\partial \mu_{2}}{\partial N_{4}}\right)_{T, p, N_{4}^{\prime}}\right], \\
& \left(\frac{\partial \mu_{3}}{\partial x_{3}}\right)_{T, p, \Sigma}=N \cdot\left[\left(\frac{\partial \mu_{3}}{\partial N_{3}}\right)_{T, p, N_{3}^{\prime}}-\left(\frac{\partial \mu_{3}}{\partial N_{4}}\right)_{T, p, N_{4}^{\prime}}\right] .
\end{aligned}
$$

For the derivative $\left(\partial \mu_{i} / \partial N_{j}\right)_{T, p, N_{j}^{\prime}}$, several auxiliary expressions were defined [16]

$$
\begin{gathered}
\Delta_{i j k}=G_{i i} G_{j j}+G_{i i} G_{k k}+G_{j j} G_{k k}+2\left(G_{i j} G_{i k}+G_{i j} G_{j k}+G_{i k} G_{j k}\right) \\
-G_{i j}^{2}-G_{i k}^{2}-G_{j k}^{2}-2\left(G_{i i} G_{j k}+G_{j j} G_{i k}+G_{k k} G_{i j}\right), i \neq j \neq k, \\
F_{i j}=G_{i i} G_{j j}-G_{i j}^{2}, i \neq j, \\
F_{i j k}=G_{i i} G_{j j} G_{k k}+2 G_{i j} G_{i k} G_{j k}-G_{i i} G_{j k}^{2}-G_{j j} G_{i k}^{2}-G_{k k} G_{i j}^{2}, i \neq j \neq k
\end{gathered}
$$

while the following three were defined in this work

$$
\begin{gathered}
\eta=\rho_{1}+\rho_{2}+\rho_{3}+\rho_{4}+\rho_{1} \rho_{2} \Delta_{12}+\rho_{1} \rho_{3} \Delta_{13}+\rho_{1} \rho_{4} \Delta_{14}+\rho_{2} \rho_{3} \Delta_{23} \\
+\rho_{2} \rho_{4} \Delta_{24}+\rho_{3} \rho_{4} \Delta_{34}+\rho_{1} \rho_{2} \rho_{3} \Delta_{123}+\rho_{1} \rho_{2} \rho_{4} \Delta_{124} \\
+\rho_{1} \rho_{3} \rho_{4} \Delta_{134}+\rho_{2} \rho_{3} \rho_{4} \Delta_{234}+\rho_{1} \rho_{2} \rho_{3} \rho_{4} \Delta_{1234} \\
F_{i i, j k, k m}=G_{i i} G_{j k} G_{k m}-G_{i j} G_{i k} G_{k m}, i \neq j \neq k \neq m,
\end{gathered}
$$

where it is important to note that matrix $\boldsymbol{G}$ is symmetric. The last auxiliary expression is

$$
\begin{aligned}
\Delta_{1234} & =F_{123}+F_{124}+F_{134}+F_{234} \\
& -2\left(G_{34} F_{12}+G_{24} F_{13}+G_{23} F_{14}+G_{14} F_{23}+G_{13} F_{24}+G_{12} F_{34}\right) \\
& +2\left(F_{11,23,34}+F_{11,24,34}+F_{11,23,24}+F_{22,14,34}+F_{22,13,34}+F_{22,13,14}\right. \\
& \left.+F_{33,14,24}+F_{33,12,24}+F_{33,12,14}+F_{44,12,13}+F_{44,12,23}+F_{44,13,23}\right) .
\end{aligned}
$$


The derivatives $\left(\partial \mu_{i} / \partial N_{j}\right)_{T, p, N_{j}^{\prime}}$ based on KBI were determined from eq. (3) [15] for quaternary mixtures

$$
\left(\frac{\partial \mu_{1}}{\partial N_{1}}\right)_{T, p, N_{1}^{\prime}}=\frac{k T\left(\rho_{2}+\rho_{3}+\rho_{4}+\rho_{2} \rho_{3} \Delta_{23}+\rho_{2} \rho_{4} \Delta_{24}+\rho_{3} \rho_{4} \Delta_{34}+\rho_{2} \rho_{3} \rho_{4} \Delta_{234}\right)}{V \rho_{1} \eta}
$$

$$
\begin{aligned}
\left(\frac{\partial \mu_{1}}{\partial N_{2}}\right)_{T, p, N_{2}^{\prime}} & =-\frac{k T}{V \eta} \cdot\left(1+\rho_{3}\left[G_{33}-G_{23}+G_{12}-G_{13}\right]+\rho_{4}\left[G_{44}-G_{14}+G_{12}-G_{24}\right]\right. \\
& +\rho_{3} \rho_{4}\left[F_{34}+G_{12} \Delta_{34}-G_{33}\left(G_{14}+G_{24}\right)-G_{44}\left(G_{13}+G_{23}\right)\right. \\
& \left.\left.+G_{23}\left(G_{14}-G_{13}+G_{34}\right)+G_{24}\left(G_{13}-G_{14}+G_{34}\right)+G_{34}\left(G_{13}+G_{14}\right)\right]\right)
\end{aligned}
$$

$$
\begin{aligned}
\left(\frac{\partial \mu_{1}}{\partial N_{3}}\right)_{T, p, N_{3}^{\prime}} & =-\frac{k T}{V \eta} \cdot\left(1+\rho_{2}\left[G_{22}-G_{12}+G_{13}-G_{23}\right]+\rho_{4}\left[G_{44}-G_{14}+G_{13}-G_{34}\right]\right. \\
& +\rho_{2} \rho_{4}\left[F_{24}+G_{13} \Delta_{24}-G_{22}\left(G_{14}+G_{34}\right)-G_{44}\left(G_{12}+G_{23}\right)\right. \\
& \left.\left.+G_{12}\left(G_{34}-G_{23}\right)+G_{14}\left(G_{23}-G_{34}\right)+G_{24}\left(G_{12}+G_{14}+G_{23}+G_{34}\right)\right]\right)
\end{aligned}
$$

$$
\begin{aligned}
\left(\frac{\partial \mu_{1}}{\partial N_{4}}\right)_{T, p, N_{4}^{\prime}} & =-\frac{k T}{V \eta} \cdot\left(1+\rho_{2}\left[G_{22}-G_{12}+G_{14}-G_{24}\right]+\rho_{3}\left[G_{33}-G_{13}+G_{14}-G_{34}\right]\right. \\
& +\rho_{2} \rho_{3}\left[F_{23}+G_{14} \Delta_{23}-G_{22}\left(G_{13}+G_{34}\right)-G_{33}\left(G_{12}+G_{24}\right)\right. \\
& \left.\left.+G_{12}\left(G_{23}-G_{24}+G_{34}\right)+G_{13}\left(G_{23}+G_{24}-G_{34}\right)+G_{23}\left(G_{24}+G_{34}\right)\right]\right)
\end{aligned}
$$

$$
\left(\frac{\partial \mu_{2}}{\partial N_{2}}\right)_{T, p, N_{2}^{\prime}}=\frac{k T\left(\rho_{1}+\rho_{3}+\rho_{4}+\rho_{1} \rho_{3} \Delta_{13}+\rho_{1} \rho_{4} \Delta_{14}+\rho_{3} \rho_{4} \Delta_{34}+\rho_{1} \rho_{3} \rho_{4} \Delta_{134}\right)}{V \rho_{2} \eta},
$$




$$
\begin{aligned}
\left(\frac{\partial \mu_{2}}{\partial N_{3}}\right)_{T, p, N_{3}^{\prime}} & =-\frac{k T}{V \eta} \cdot\left(1+\rho_{1}\left[G_{11}-G_{12}+G_{23}-G_{13}\right]+\rho_{4}\left[G_{44}-G_{24}+G_{23}-G_{34}\right]\right. \\
& +\rho_{1} \rho_{4}\left[F_{14}+G_{23} \Delta_{14}-G_{11}\left(G_{24}+G_{34}\right)-G_{44}\left(G_{12}+G_{13}\right)\right. \\
& \left.\left.+G_{12}\left(G_{14}-G_{13}+G_{34}\right)+G_{14}\left(G_{13}+G_{24}+G_{34}\right)+G_{24}\left(G_{13}-G_{34}\right)\right]\right)
\end{aligned}
$$

$$
\begin{aligned}
\left(\frac{\partial \mu_{2}}{\partial N_{4}}\right)_{T, p, N_{4}^{\prime}} & =-\frac{k T}{V \eta} \cdot\left(1+\rho_{1}\left[G_{11}-G_{12}+G_{24}-G_{14}\right]+\rho_{3}\left[G_{33}-G_{23}+G_{24}-G_{34}\right]\right. \\
& +\rho_{1} \rho_{3}\left[F_{13}+G_{24} \Delta_{13}-G_{11}\left(G_{23}+G_{34}\right)-G_{33}\left(G_{12}+G_{14}\right)\right. \\
& \left.\left.+G_{12}\left(G_{13}-G_{14}+G_{34}\right)+G_{13}\left(G_{14}+G_{23}+G_{34}\right)+G_{23}\left(G_{14}-G_{34}\right)\right]\right)
\end{aligned}
$$

$$
\left(\frac{\partial \mu_{3}}{\partial N_{3}}\right)_{T, p, N_{3}^{\prime}}=\frac{k T\left(\rho_{1}+\rho_{2}+\rho_{4}+\rho_{1} \rho_{2} \Delta_{12}+\rho_{1} \rho_{4} \Delta_{14}+\rho_{2} \rho_{4} \Delta_{24}+\rho_{1} \rho_{2} \rho_{4} \Delta_{124}\right)}{V \rho_{3} \eta}
$$

$$
\begin{aligned}
\left(\frac{\partial \mu_{3}}{\partial N_{4}}\right)_{T, p, N_{4}^{\prime}} & =-\frac{k T}{V \eta} \cdot\left(1+\rho_{1}\left[G_{11}-G_{13}+G_{34}-G_{14}\right]+\rho_{2}\left[G_{22}-G_{23}+G_{34}-G_{24}\right]\right. \\
& +\rho_{1} \rho_{2}\left[F_{12}+G_{34} \Delta_{12}-G_{11}\left(G_{23}+G_{24}\right)-G_{22}\left(G_{13}+G_{14}\right)\right. \\
& \left.\left.+G_{12}\left(G_{13}+G_{23}+G_{14}+G_{24}\right)+G_{13}\left(G_{24}-G_{14}\right)+G_{23}\left(G_{14}-G_{24}\right)\right]\right) .
\end{aligned}
$$

To determine the derivatives $\left(\partial \mu_{i} / \partial x_{j}\right)_{T, p, \Sigma}$, eqs. (C1) to $(\mathrm{C} 9)$ were combined with eqs. (C16) to (C24) and the thermodynamic factor elements of $\boldsymbol{\Gamma}$ for a quaternary mixture are obtained by applying eq. (1)

$$
\begin{aligned}
\Gamma_{11} & =\frac{1}{\eta} \cdot\left(\rho_{1}+\rho_{2}+\rho_{3}+\rho_{4}+\rho_{2} \rho_{3} \Delta_{23}+\rho_{2} \rho_{4} \Delta_{24}+\rho_{3} \rho_{4} \Delta_{34}+\rho_{2} \rho_{3} \rho_{4} \Delta_{234}\right. \\
& +\rho_{1} \rho_{2}\left[G_{22}-G_{12}+G_{14}-G_{24}\right]+\rho_{1} \rho_{3}\left[G_{33}-G_{13}+G_{14}-G_{34}\right] \\
& +\rho_{1} \rho_{2} \rho_{3}\left[F_{23}+G_{14} \Delta_{23}-G_{22}\left(G_{13}+G_{34}\right)-G_{33}\left(G_{12}+G_{24}\right)\right. \\
& \left.\left.+G_{12}\left(G_{23}-G_{24}+G_{34}\right)+G_{13}\left(G_{23}+G_{24}-G_{34}\right)+G_{23}\left(G_{24}+G_{34}\right)\right]\right),
\end{aligned}
$$




$$
\begin{aligned}
\Gamma_{21} & =\frac{\rho_{2}}{\eta} \cdot\left(\rho_{1}\left[G_{11}-G_{12}+G_{24}-G_{14}\right]+\rho_{3}\left[G_{24}-G_{34}+G_{13}-G_{12}\right]\right. \\
& -\rho_{4}\left[G_{44}-G_{14}+G_{12}-G_{24}\right]+\rho_{1} \rho_{3}\left[F_{13}+G_{24} \Delta_{13}-G_{11}\left(G_{23}+G_{34}\right)\right. \\
& -G_{33}\left(G_{12}+G_{14}\right)+G_{12}\left(G_{13}-G_{14}+G_{34}\right)+G_{13}\left(G_{14}+G_{23}+G_{34}\right) \\
& \left.+G_{23}\left(G_{14}-G_{34}\right)\right]-\rho_{3} \rho_{4}\left[F_{34}+G_{12} \Delta_{34}-G_{33}\left(G_{14}+G_{24}\right)\right. \\
& -G_{44}\left(G_{13}+G_{23}\right)+G_{23}\left(G_{14}-G_{13}+G_{34}\right)+G_{24}\left(G_{13}-G_{14}+G_{34}\right) \\
& \left.\left.+G_{34}\left(G_{13}+G_{14}\right)\right]\right),
\end{aligned}
$$

$$
\begin{aligned}
\Gamma_{31} & =\frac{\rho_{3}}{\eta} \cdot\left(\rho_{1}\left[G_{11}-G_{13}+G_{34}-G_{14}\right]+\rho_{2}\left[G_{34}-G_{24}+G_{12}-G_{13}\right]\right. \\
& -\rho_{4}\left[G_{44}-G_{14}+G_{13}-G_{34}\right]+\rho_{1} \rho_{2}\left[F_{12}+G_{34} \Delta_{12}-G_{11}\left(G_{23}+G_{24}\right)\right. \\
& -G_{22}\left(G_{13}+G_{14}\right)+G_{12}\left(G_{13}+G_{23}+G_{14}+G_{24}\right)+G_{13}\left(G_{24}-G_{14}\right) \\
& \left.+G_{23}\left(G_{14}-G_{24}\right)\right]-\rho_{2} \rho_{4}\left[F_{24}+G_{13} \Delta_{24}-G_{22}\left(G_{14}+G_{34}\right)\right. \\
& -G_{44}\left(G_{12}+G_{23}\right)+G_{12}\left(G_{34}-G_{23}\right)+G_{14}\left(G_{23}-G_{34}\right) \\
& \left.\left.+G_{24}\left(G_{12}+G_{14}+G_{23}+G_{34}\right)\right]\right),
\end{aligned}
$$

$$
\begin{aligned}
\Gamma_{12} & =\frac{\rho_{1}}{\eta} \cdot\left(\rho_{2}\left[G_{22}-G_{12}+G_{14}-G_{24}\right]+\rho_{3}\left[G_{14}-G_{34}+G_{23}-G_{12}\right]\right. \\
& -\rho_{4}\left[G_{44}-G_{14}+G_{12}-G_{24}\right]+\rho_{2} \rho_{3}\left[F_{23}+G_{14} \Delta_{23}-G_{22}\left(G_{13}+G_{34}\right)\right. \\
& -G_{33}\left(G_{12}+G_{24}\right)+G_{12}\left(G_{23}-G_{24}+G_{34}\right)+G_{13}\left(G_{23}+G_{24}-G_{34}\right) \\
& \left.+G_{23}\left(G_{24}+G_{34}\right)\right]-\rho_{3} \rho_{4}\left[F_{34}+G_{12} \Delta_{34}-G_{33}\left(G_{14}+G_{24}\right)\right. \\
& -G_{44}\left(G_{13}+G_{23}\right)+G_{23}\left(G_{14}-G_{13}+G_{34}\right)+G_{24}\left(G_{13}-G_{14}+G_{34}\right) \\
& \left.\left.+G_{34}\left(G_{13}+G_{14}\right)\right]\right)
\end{aligned}
$$

$$
\begin{aligned}
\Gamma_{22} & =\frac{1}{\eta} \cdot\left(\rho_{1}+\rho_{2}+\rho_{3}+\rho_{4}+\rho_{1} \rho_{3} \Delta_{13}+\rho_{1} \rho_{4} \Delta_{14}+\rho_{3} \rho_{4} \Delta_{34}+\rho_{1} \rho_{3} \rho_{4} \Delta_{134}\right. \\
& +\rho_{1} \rho_{2}\left[G_{11}-G_{12}+G_{24}-G_{14}\right]+\rho_{2} \rho_{3}\left[G_{33}-G_{23}+G_{24}-G_{34}\right] \\
& +\rho_{1} \rho_{2} \rho_{3}\left[F_{13}+G_{24} \Delta_{13}-G_{11}\left(G_{23}+G_{34}\right)-G_{33}\left(G_{12}+G_{14}\right)\right. \\
& \left.\left.+G_{12}\left(G_{13}-G_{14}+G_{34}\right)+G_{13}\left(G_{14}+G_{23}+G_{34}\right)+G_{23}\left(G_{14}-G_{34}\right)\right]\right),
\end{aligned}
$$




$$
\begin{aligned}
\Gamma_{32} & =\frac{\rho_{3}}{\eta} \cdot\left(\rho_{1}\left[G_{34}-G_{14}+G_{12}-G_{23}\right]+\rho_{2}\left[G_{22}-G_{23}+G_{34}-G_{24}\right]\right. \\
& -\rho_{4}\left[G_{44}-G_{24}+G_{23}-G_{34}\right]+\rho_{1} \rho_{2}\left[F_{12}+G_{34} \Delta_{12}-G_{11}\left(G_{23}+G_{24}\right)\right. \\
& -G_{22}\left(G_{13}+G_{14}\right)+G_{12}\left(G_{13}+G_{23}+G_{14}+G_{24}\right)+G_{13}\left(G_{24}-G_{14}\right) \\
& \left.+G_{23}\left(G_{14}-G_{24}\right)\right]-\rho_{1} \rho_{4}\left[F_{14}+G_{23} \Delta_{14}-G_{11}\left(G_{24}+G_{34}\right)\right. \\
& -G_{44}\left(G_{12}+G_{13}\right)+G_{12}\left(G_{14}-G_{13}+G_{34}\right)+G_{14}\left(G_{13}+G_{24}+G_{34}\right) \\
& \left.\left.+G_{24}\left(G_{13}-G_{34}\right)\right]\right),
\end{aligned}
$$

$$
\begin{aligned}
\Gamma_{13} & =\frac{\rho_{1}}{\eta} \cdot\left(\rho_{2}\left[G_{14}-G_{24}+G_{23}-G_{13}\right]+\rho_{3}\left[G_{33}-G_{13}+G_{14}-G_{34}\right]\right. \\
& -\rho_{4}\left[G_{44}-G_{14}+G_{13}-G_{34}\right]+\rho_{2} \rho_{3}\left[F_{23}+G_{14} \Delta_{23}-G_{22}\left(G_{13}+G_{34}\right)\right. \\
& -G_{33}\left(G_{12}+G_{24}\right)+G_{12}\left(G_{23}-G_{24}+G_{34}\right)+G_{13}\left(G_{23}+G_{24}-G_{34}\right) \\
& \left.+G_{23}\left(G_{24}+G_{34}\right)\right]-\rho_{2} \rho_{4}\left[F_{24}+G_{13} \Delta_{24}-G_{22}\left(G_{14}+G_{34}\right)\right. \\
& -G_{44}\left(G_{12}+G_{23}\right)+G_{12}\left(G_{34}-G_{23}\right)+G_{14}\left(G_{23}-G_{34}\right) \\
& \left.\left.+G_{24}\left(G_{12}+G_{14}+G_{23}+G_{34}\right)\right]\right),
\end{aligned}
$$

$$
\begin{aligned}
\Gamma_{23} & =\frac{\rho_{2}}{\eta} \cdot\left(\rho_{1}\left[G_{24}-G_{14}+G_{13}-G_{23}\right]+\rho_{3}\left[G_{33}-G_{23}+G_{24}-G_{34}\right]\right. \\
& -\rho_{4}\left[G_{44}-G_{24}+G_{23}-G_{34}\right]+\rho_{1} \rho_{3}\left[F_{13}+G_{24} \Delta_{13}-G_{11}\left(G_{23}+G_{34}\right)\right. \\
& -G_{33}\left(G_{12}+G_{14}\right)+G_{12}\left(G_{13}-G_{14}+G_{34}\right)+G_{13}\left(G_{14}+G_{23}+G_{34}\right) \\
& \left.+G_{23}\left(G_{14}-G_{34}\right)\right]-\rho_{1} \rho_{4}\left[F_{14}+G_{23} \Delta_{14}-G_{11}\left(G_{24}+G_{34}\right)\right. \\
& -G_{44}\left(G_{12}+G_{13}\right)+G_{12}\left(G_{14}-G_{13}+G_{34}\right)+G_{14}\left(G_{13}+G_{24}+G_{34}\right) \\
& \left.\left.+G_{24}\left(G_{13}-G_{34}\right)\right]\right)
\end{aligned}
$$

$$
\begin{aligned}
\Gamma_{33} & =\frac{1}{\eta} \cdot\left(\rho_{1}+\rho_{2}+\rho_{3}+\rho_{4}+\rho_{1} \rho_{2} \Delta_{12}+\rho_{1} \rho_{4} \Delta_{14}+\rho_{2} \rho_{4} \Delta_{24}+\rho_{1} \rho_{2} \rho_{4} \Delta_{124}\right. \\
& +\rho_{1} \rho_{3}\left[G_{11}-G_{13}+G_{34}-G_{14}\right]+\rho_{2} \rho_{3}\left[G_{22}-G_{23}+G_{34}-G_{24}\right] \\
& +\rho_{1} \rho_{2} \rho_{3}\left[F_{12}+G_{34} \Delta_{12}-G_{11}\left(G_{23}+G_{24}\right)-G_{22}\left(G_{13}+G_{14}\right)\right. \\
& \left.\left.+G_{12}\left(G_{13}+G_{23}+G_{14}+G_{24}\right)+G_{13}\left(G_{24}-G_{14}\right)+G_{23}\left(G_{14}-G_{24}\right)\right]\right) .
\end{aligned}
$$

The pure fluid limits are equivalent to the binary and ternary cases, except for their dimension, 


$$
\lim _{\substack{x_{1} \rightarrow 0 \\
x_{2} \rightarrow 0 \\
x_{3} \rightarrow 0}} \boldsymbol{\Gamma}=\lim _{\substack{x_{2} \rightarrow 0 \\
x_{3} \rightarrow 0 \\
x_{4} \rightarrow 0}} \boldsymbol{\Gamma}=\lim _{\substack{x_{1} \rightarrow 0 \\
x_{3} \rightarrow 0 \\
x_{4} \rightarrow 0}} \boldsymbol{\Gamma}=\lim _{\substack{x_{1} \rightarrow 0 \\
x_{2} \rightarrow 0 \\
x_{4} \rightarrow 0}} \boldsymbol{\Gamma}=\left(\begin{array}{ccc}
1 & 0 & 0 \\
0 & 1 & 0 \\
0 & 0 & 1
\end{array}\right)
$$

The second limit, i.e. quaternary mixture towards its binary subsystem, is

$$
\begin{aligned}
& \lim _{\substack{x_{1} \rightarrow 0 \\
x_{2} \rightarrow 0}} \boldsymbol{\Gamma}=\left(\begin{array}{ccc}
1 & 0 & 0 \\
0 & 1 & 0 \\
\Gamma_{31} & \Gamma_{32} & \Gamma_{B}
\end{array}\right), \\
& \lim _{\substack{x_{1} \rightarrow 0 \\
x_{3} \rightarrow 0}} \boldsymbol{\Gamma}=\left(\begin{array}{ccc}
1 & 0 & 0 \\
\Gamma_{21} & \Gamma_{B} & \Gamma_{23} \\
0 & 0 & 1
\end{array}\right), \\
& \lim _{\substack{x_{2} \rightarrow 0 \\
x_{3} \rightarrow 0}} \boldsymbol{\Gamma}=\left(\begin{array}{ccc}
\Gamma_{B} & \Gamma_{12} & \Gamma_{13} \\
0 & 1 & 0 \\
0 & 0 & 1
\end{array}\right),
\end{aligned}
$$

$$
\lim _{\substack{x_{3} \rightarrow 0 \\
x_{4} \rightarrow 0}} \boldsymbol{\Gamma}=\left(\begin{array}{ccc}
\Gamma_{B}+\Gamma_{12} & \Gamma_{12} & \Gamma_{13} \\
\Gamma_{21} & \Gamma_{B}+\Gamma_{21} & \Gamma_{23} \\
0 & 0 & 1
\end{array}\right)
$$

Note that all $\Gamma_{i j}$ in eqs. (C35) to (C40) correspond to eqs. (C25) to (C33). For instance, $x_{1} \rightarrow 0$ and $x_{2} \rightarrow 0$, results in main elements $\Gamma_{11}=1$ and $\Gamma_{22}=1$. The other matrix cross elements of the first two lines are zero. The last matrix line with the cross 
elements $\Gamma_{3 j}$ implies solutions that are neither zero nor unity because of $x_{3} \rightarrow 1$. These elements $\Gamma_{31}$ and $\Gamma_{32}$ correspond to eqs. (C27) and (C30) and for $x_{1} \rightarrow 0$, $x_{2} \rightarrow 0$ parts of those equations vanish, however, a statistically sound sampling with KBI is not straightforward because RDF $g_{13}, g_{14}, g_{23}$ and $g_{24}$ are needed at infinite dilution of components 1 and 2. The main element $\Gamma_{B}$ is the binary factor of the system $3+4$ given by eq. (A1). The other cases are analogous.

The last limit, i.e. quaternary mixture towards its ternary subsystems, is

$$
\begin{aligned}
\lim _{x_{1} \rightarrow 0} \boldsymbol{\Gamma} & =\left(\begin{array}{ccc}
1 & 0 & 0 \\
\Gamma_{21} & \Gamma_{T, 11} & \Gamma_{T, 12} \\
\Gamma_{31} & \Gamma_{T, 21} & \Gamma_{T, 22}
\end{array}\right), \\
\lim _{x_{2} \rightarrow 0} \boldsymbol{\Gamma} & =\left(\begin{array}{ccc}
\Gamma_{T, 11} & \Gamma_{12} & \Gamma_{T, 12} \\
0 & 1 & 0 \\
\Gamma_{T, 21} & \Gamma_{32} & \Gamma_{T, 22}
\end{array}\right), \\
\lim _{x_{3} \rightarrow 0} \boldsymbol{\Gamma} & =\left(\begin{array}{ccc}
\Gamma_{T, 11} & \Gamma_{T, 12} & \Gamma_{13} \\
\Gamma_{T, 21} & \Gamma_{T, 22} & \Gamma_{23} \\
0 & 0 & 1
\end{array}\right), \\
\lim _{x_{4} \rightarrow 0} \boldsymbol{\Gamma} & =\left(\begin{array}{ccc}
\Gamma_{T, 11}+\Gamma_{13} & \Gamma_{T, 12}+\Gamma_{13} & \Gamma_{13} \\
\Gamma_{T, 21}+\Gamma_{23} & \Gamma_{T, 22}+\Gamma_{23} & \Gamma_{23} \\
\Gamma_{31} & \Gamma_{32} & \Gamma_{33}
\end{array}\right),
\end{aligned}
$$

Looking at eq. (C41), the first matrix line containing the elements $\Gamma_{1 j}$ is clear from $x_{1} \rightarrow 0$ in eqs. (C25), (C28) and (C31). The elements $\Gamma_{21} \neq 0$ and $\Gamma_{31} \neq 0$ correspond to eqs. (C26) and (C27). For $x_{1} \rightarrow 0$, parts of the latter two equations vanish, but a statistically sound sampling with KBI is not straightforward because RDF $g_{12}, g_{13}$ and $g_{14}$ are needed at infinite dilution of component 1 . The remaining elements $\Gamma_{T, i j}$ constitute the thermodynamic factor of the ternary mixture $2+3+4$, cf. eqs. (B3) to (B6). The other cases are analogous. 